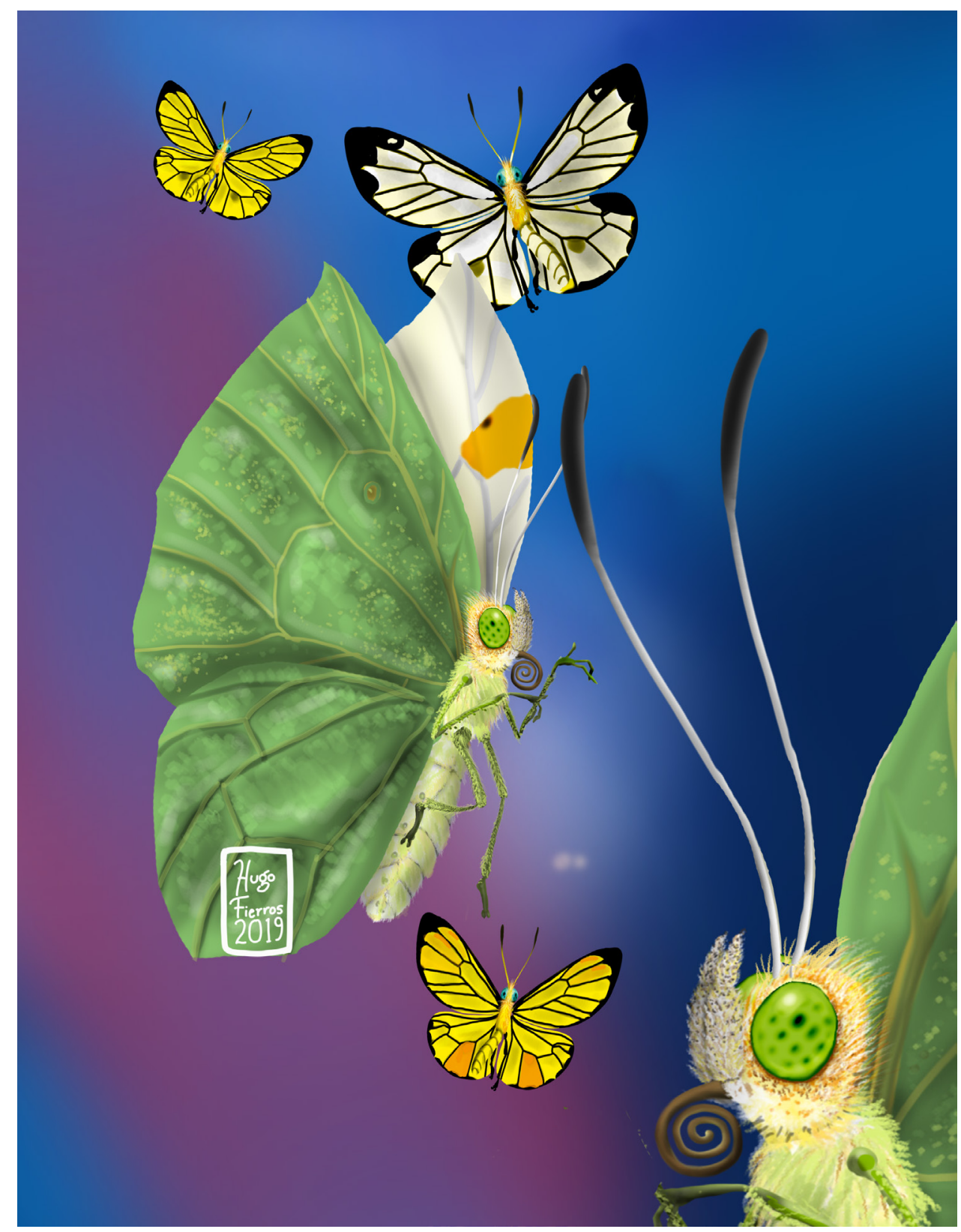

Dugesiana, Año 26, No. 2, julio 2019-diciembre 2019 (segundo semestre de 2019), es una publicación semestral, editada por la Universidad de Guadalajara, a través del Centro de Estudios en Zoología, por el Centro Universitario de Ciencias Biológicas y Agropecuarias. Camino Ramón Padilla Sánchez \# 2100, Nextipac, Zapopan, Jalisco, Tel. 37771150 ext. 33218, http://148.202.248.171/dugesiana/index.php/DUG/index, glenusmx@gmail.com. Editor responsable: José Luis Navarrete Heredia. Reserva de Derechos al Uso Exclusivo 04-2009-062310115100203, ISSN: 2007-9133, otorgados por el Instituto Nacional del Derecho de Autor. Responsable de la última actualización de este número: José Luis Navarrete Heredia, Editor y Ana Laura González-Hernández, Asistente Editorial. Fecha de la última modificación 25 de julio 2019, con un tiraje de un ejemplar.

Las opiniones expresadas por los autores no necesariamente reflejan la postura del editor de la publicación.

Queda estrictamente prohibida la reproducción total o parcial de los contenidos e imágenes de la publicación sin previa autorización de la Universidad de Guadalajara. 


\title{
Lista comentada de las hormigas (Hymenoptera: Formicidae) del cerro Juan El Grande, El Llano, Aguascalientes, México
}

\section{Checklist of ants (Hymenoptera: Formicidae) of Juan El Grande hill, El Llano, Aguascalientes, Mexico}

\section{Emmanuel González-Martínez ${ }^{1}$ y Miguel Vásquez-Bolaños ${ }^{2 *}$}

${ }^{1}$ Colección Zoológica, Departamento de Biología, Centro de Ciencias Básicas, Universidad Autónoma de Aguascalientes. Avenida Universidad \# 940, Ciudad Universitaria, Aguascalientes, Aguascalientes, México, C. P. 20130, biol.emmanuel.glz.mtz@gmail.com; 2Entomología, Centro de Estudios en Zoología, Centro Universitario de Ciencias Biológicas y Agropecuarias, Universidad de Guadalajara. Ramón Padilla Sánchez \# 2100, Las Agujas, Zapopan, Jalisco, México, C. P. 45220.mvb14145@hotmail.com

\section{RESUMEN}

No obstante de ser organismos muy importantes en los ecosistemas terrestres, poco se han estudiado las hormigas en el estado de Aguascalientes. El cerro Juan El Grande se ubica en el municipio de El Llano, presenta un gradiente de vegetación de bosque de encino arbustivo abierto en las partes altas y laderas y asociaciones principalmente de jarilla en las partes bajas; rodeado de campos de cultivo y áreas de pastoreo. Se colectó en dos sitios: bosque de encino y vegetación secundaria, de noviembre de 2016 a julio de 2017 , con trampas de caída, muestras de hojarasca y colecta manual; se realizaron cuatro muestreos: otoño, invierno, primavera y verano. Se obtuvieron 4,815 individuos, agrupados en 26 especies, 15 géneros, 12 tribus y cinco subfamilias. Se elaboró una lista comentada, con un diagnosis y distribución para cada género; para las especies encontradas se incluye número de individuos y castas; temporada, sitio (tipo de vegetación) y método de colecta; así como distribución. La vegetación con mayor riqueza fue bosque de encino, con 20 especies y vegetación secundaria con 16 especies. La temporada con mayor riqueza fue verano con 24 especies, primavera con 20 ; otoño con 16 e invierno con 13 especies. El método de colecta con mayor riqueza fueron las trampas cebadas con 24 especies, seguido de colecta directa con 18 especies y por último el cernido de hojarasca con tres especies. En el área de estudio se encontró una cantidad considerable de especies de hormigas a pesar de presentar perturbación, es necesario realizar más estudios relacionados con este grupo en el estado de Aguascalientes, a fin de contar con listados lo más completos posibles sobre diversidad.

Palabras clave: Riqueza específica, lista comentada, Altiplano.

\begin{abstract}
Ants are very important organisms in terrestrial ecosystems, although little has been studied in the Aguascalientes state. The hill Juan El Grande is located in El Llano municipality, with a vegetation gradient of oak forest in the highlands and slopes and associations of jarilla in lowlands. Juan El Grande is surrounded by crop fields and grazing areas. The collect was in two sites: oak forest and disturbed vegetation, from November 2016 to July 2017, pitfall and carriontraps, leaflitter samples and manual collection were used; four samplings were carried out: autumn, winter, spring and summer. There were 4,815 individuals, into 26 species, 15 genera, 12 tribes and 5 subfamilies. A checklist of Juan El Grande hill was elaborated, diagnosis and distribution for each genus; and for each ant species are provided specimens and castes; season, site (vegetation) and collection method; and distribution. The vegetation with the greatest richness was oak forest, 20 species and secondary vegetation with 16 species. The season with more species was summer with 24 species, spring with 20; autumn with 16 and winter with 13 species. The method of collection with the greatest richness was pitfall with 24 species, followed by direct collection with 18 species and finally the leaf-litter with 3 species. In the area studied, despite having disturbance, a considerable number of ant species was collected; it is necessary to development more studies of this group in Aguascalientes state, for to have list as complete as possible about diversity of ants.
\end{abstract}

Key words: Specific richness, Checklist, Altiplano.

Todas las especies de hormigas se agrupan en la familia Formicidae, dentro de la superfamilia Vespoidea, del orden Hymenoptera. Llamados insectos eusociales, cuentan con: traslape de generaciones como adultos, castas diferenciadas reinas y machos (función reproductiva) generalmente presentan alas; las obreras (hembras estériles) son ápteras (Jaffé 1993; Rojas-Fernández 2001). Las principales características morfológicas que presentan son: Cabeza ortognata con dos ojos compuestos, en ocasiones hasta tres ocelos; antenas geniculadas; aparato bucal masticador- lamedor, modificado en soldados y en especies parásitas. Mesosoma: formado por la fusión del tórax y el primer segmento del abdomen (propodeo); en la parte ventral tres pares de patas y en la parte dorsal dos pares de alas membranosas, sólo en los reproductores; en el propodeo tienen la abertura de la glándula metapleural. Peciolo: segundo segmento abdominal diferenciado y articulado. Pospeciolo: es el tercer segmento abdominal diferenciado y articulado, presente en algunas subfamilias de hormigas. Gáster: el resto del abdomen, contiene el estómago social, 
órganos reproductores y un aguijón (sólo en hembras) en la mayoría de las especies (Vásquez-Bolaños y Quiroz-Rocha 2013).

La familia Formicidae está dividida en 20 subfamilias, 16 con representantes vivos y cuatro conocidas sólo a partir de fósiles, se han descrito casi 15,000 especies en todo el mundo, siendo los trópicos los lugares con la mayor cantidad de especies y de endemismos. Se reportan 927 especies de hormigas para México, pertenecen a 93 géneros y 11 subfamilias, las cuales se encuentran distribuidas en todos los estados de la República incluidas algunas islas, aunque la mayoría se concentran en el sureste del país (Vásquez-Bolaños 2015).

Están presentes en todos los ecosistemas terrestres, excepto en los polos y los glaciares (Jaffé 1993), se localizan en los bosques tropicales, bosques templados, zonas áridas y semiáridas, áreas urbanas y agroecosistemas (Vásquez-Bolaños y Quiroz-Rocha 2013). En los trópicos se encuentran la mayor riqueza de especies y abundancia, se distribuyen desde el nivel del mar hasta los 4,000 metros de altitud (Vásquez-Bolaños 2011). Habitan desde el subsuelo hasta las copas de los árboles, son habitantes del suelo por excelencia, la mayoría de las especies viven en nidos subterráneos, en la hojarasca o en la madera en descomposición depositada sobre el suelo, aunque algunas especies anidan en plantas o dentro de ellas (Rojas-Fernández 2001). Los hábitos alimentarios van desde depredadoras, herbívoras o detritívoras, algunas son cortadoras de hojas que utilizan para cultivar hongos, granívoras, granjeras y necrófagas (Vásquez Bolaños 1998). Representan uno de los grupos terrestres más abundantes, se calcula que hay $19^{15}$ de hormigas vivas sobre la tierra (Wilson 1971). El número de hormigas de una colonia puede ser de unas cuantas docenas hasta alcanzar los 300 millones de obreras (Vásquez Bolaños 1998), por lo tanto, la abundancia real de este grupo es difícil de medir ya que la mayoría de los individuos de una colonia se encuentran dentro del nido y los que salen a forrajear lo hacen a intervalos de tiempo limitados (Rojas-Fernández 2001). Para el caso de las hormigas la abundancia y riqueza de especies disminuye conforme se incrementa la altitud y latitud, debido a su relación con factores bióticos y abióticos (SalcidoLópez et al. 2012). En las zonas áridas y semiáridas, las hormigas son un componente faunístico importante por la alta riqueza de especies y endemismos que se presentan, por las interacciones biológicas que establecen con otros organismos, la remoción y consumo de semillas (HinojosaOntiveros et al. 2015). Son de gran importancia, debido a su papel en los ecosistemas terrestres, además son utilizados como indicadores biológicos, mantienen interacciones muy importantes con plantas y diversos organismos, algunas hormigas poseen una importancia alimenticia y en la salud humana al producir reacciones alérgicas por su picadura o mordedura (Fernández 2003).

Aun son escasos los estudios taxonómicos de hormigas en México, a pesar de la importancia del grupo y de la compleja diversidad del país dada su ubicación, ya que incluye la zona de transición entre las regiones Neártica y Neotropical, teniendo especies representantes de ambas regiones, así como propias (Vásquez-Bolaños 2011). Vásquez-Bolaños (2013) menciona que para México el primer trabajo en español sobre hormigas mexicanas fue el de Edward Norton publicado en 1875, en la revista La Naturaleza, traducción del publicado en 1868 en inglés (Norton 1875). Por otra parte, Mackay y Mackay (1989) incluyen una clave ilustrada para los géneros de hormigas de México y un listado de los mismos. Para México, los trabajos que destacan son: Rojas-Fernández (1996) en donde aporta un primer inventario para las especies de hormigas de todo México con información por estado, reporta 501 especies; en 2001 la misma autora presenta un estudio y una lista de especies de hormigas que habitan en el suelo y la hojarasca en México; acerca de las hormigas del norte de México; Ríos-Casanova et al. (2004) mencionan que el conocimiento de las hormigas que habitan en las zonas áridas y semiáridas en México es escaso ya que únicamente existen tres estudios al respecto. Alatorre-Bracamontes y Vásquez-Bolaños (2010) integran una lista comentada para el norte del país en donde reportan 290 especies, 10 de estas especies son nuevos registros para México; en 2011 Vásquez-Bolaños presenta una lista de especies de hormigas para México, con base en revisión literaria, con un total de 884 especies de hormigas pertenecientes a 86 géneros, 33 tribus y once subfamilias, en donde menciona que el estado con mayor especies es Veracruz con 283 y los estados que registran la menor cantidad de especies son Tlaxcala con dos, Aguascalientes con nueve. Para el 2015 se presenta una lista actualizada de las especies de hormigas para México en donde Vásquez-Bolaños (2015) reporta 927 especies pertenecientes a 93 géneros y 11 subfamilias.

Para el estado de Aguascalientes los antecedentes de la familia Formicidae son una tesis de licenciatura realizada por Cañedo-Ortiz (1988) para el municipio de Aguascalientes, donde registra ocho géneros y nueve especies; una investigación sobre biodiversidad de himenópteros para el estado realizado por Escoto-Rocha et al. (2001) en la cual se identificaron siete especies de hormigas y tres a nivel de género; para el estado de Aguascalientes VásquezBolaños y Cisneros-Caballero (2013) reportan 25 especies; En un trabajo de revisión de la Colección Zoológica del Departamento de Biología de la Universidad Autónoma de Aguascalientes realizado por Rodríguez-Elizalde y Escoto-Rocha (2015) reportan 17 especies para el estado. El último trabajo que se tiene para Aguascalientes es una lista de especies de hormigas realizado por VásquezBolaños y Escoto-Rocha (2016), con base en los ejemplares depositados en las colecciones entomológicas de la Universidad Autónoma de Aguascalientes y de la Universidad de Guadalajara, en donde además se tomó en cuenta en publicaciones especializadas sobre la familia Formicidae para el estado de Aguascalientes, en donde se tienen 32 especies de hormigas, siendo el municipio de 
Aguascalientes en el que se conoce mayor número, con 19 especies. En cuanto a los municipios de Cosío, El Llano, Rincón de Romos y Tepezalá no se tienen especies de hormigas registradas.

Los estudios sobre la familia Formicidae en el estado de Aguascalientes en general son escasos, son contados los trabajos que aborden este grupo y la gran mayoría del estado no ha sido explorada, específicamente para el caso del municipio de El Llano. Para conocer que especies de hormigas están en el área de estudio, lo que servirá en un futuro como base para diversos estudios referentes a estos organismos para el municipio y el estado, el objetivo es conocer la riqueza de especies y elaborar una lista comentada de las hormigas del cerro Juan El Grande, en el municipio de El Llano, Aguascalientes.

\section{MATERIALES Y MÉTODOS}

El cerro Juan El Grande se ubica en el ejido de Palo Alto dentro del municipio de El Llano, en el este de estado de Aguascalientes, en las coordenadas $21^{\circ} 56^{\prime} 19^{\prime \prime}$ latitud norte y $101^{\circ} 55^{\prime} 4$ "' longitud oeste, presenta una gradiente altitudinal desde los 2,500 hasta los 2,090 m snm. Se encuentra en la provincia fisiográfica de la Mesa Central y en la subprovincia Llanuras de Ojuelos-Aguascalientes; esta subprovincia se caracteriza por presentar llanos extensos situados entre 2,000 y 2,500 m snm de piso consolidado y cubierto sólo por una capa de aluviones (referencia). En cuanto su sistema de topoforma presenta relieve tipo Meseta, el clima es semiseco templado (BS1k). Presenta una temperatura promedio anual es de $17.1^{\circ} \mathrm{C}$, las temperaturas más bajas se presentan en enero con $12.6^{\circ} \mathrm{C}$ en promedio, mientras que las más altas son en mayo con $28.8^{\circ} \mathrm{C}$ en promedio. El periodo de lluvias se presenta en verano con una precipitación media anual de $480 \mathrm{~mm}$, el mes más lluvioso en promedio es julio con $120 \mathrm{~mm}$. Su geología está constituida por rocas ígneas de extrusiones terciarias tipo riolita-toba ácida y de la era del cuaternario con rocas ígneas extrusivas tipo basalto (Fig. 1). El tipo de suelo es xerosol háplico que son suelos que se caracterizan por presentar una capa superficial de color claro y muy pobre en humus (INEGI 2001, 2009, 2015). Presenta una vegetación en las partes altas y laderas de bosque de encino arbustivo abierto, las especies de encino más abundante son Quercus microphylla Neé y $Q$. eduardi Trel., en sus partes bajas se encuentra con vegetación secundaria arbustiva y herbácea en la que predomina la jarilla (Dodonaea viscosa L. Jacq.). La zona de estudio queda incluida dentro del Área de Protección del Águila Real de la serranía de Juan Grande, Área Natural Protegida por la CONANP y la SEMARNAT (SUBECO 2001).

Se eligieron dos sitios con diferente tipo de vegetación: bosque de encino (BE) y vegetación secundaria (VS). Cada sitio fue visitado en cuatro ocasiones durante un año, en noviembre de 2016, enero, abril y julio de 2017 que representan las estaciones de otoño, invierno, primavera y verano respectivamente. Para cada sitio se eligieron dos puntos, en cada punto se colocaron cinco trampas, cuatro de caída: cebadas con fruta (TF), atún (TA), miel (TM), nuez (TN) y una necrotrampa cebada con calamar (NTP80); las trampas enterradas a nivel del suelo, con alcohol $70 \%$ como conservador y vinagre blanco para evitar su evaporación, permanecieron activas durante 30 días. Se recolectaron 2 muestras de cernido de hojarasca $(\mathrm{CH})$ de un metro cuadrado, este método sólo para aquellos sitios con hojarasca, se tamizó y se procesó en el saco Winkler. El método de colecta directa (CD), se realizó con la ayuda de pinzas entomológicas y pinceles, se buscó entre la vegetación, debajo de rocas, entre la hojarasca, troncos, cada vez que se visitó el lugar. Es recomendable usar varios métodos de colecta para tener representada la mayoría de las especies del lugar de estudio (Alonso y Agosti 2000; Sarmiento-M. 2003). Las muestras se colocaron en frascos con alcohol al $70 \%$ para su preservación, previamente etiquetados con datos básicos de campo, se trasladaron al laboratorio de la Colección Zoológica del Departamento de Biología de la Universidad Autónoma de Aguascalientes (CZUAA), donde se llevó a acabo la separación, montaje, determinación y etiquetado; para la determinación se utilizaron las claves especializadas para el grupo (Francoeur 1973, Del Toro et al. 2009, MacGown et al. 2014, Rabeling et al. 2007, Mackay y Mackay 1989, Bolton 1994). El material se encuentra depositado en la Colección Zoológica del Departamento de Biología de la Universidad Autónoma de Aguascalientes (CZUAA) y en la Colección Entomológica del Centro de Estudios en Zoología de la Universidad de Guadalajara (CZUG).

Los ejemplares de hormigas fueron etiquetados con la información de colecta: país, estado, municipio, localidad, sitio, coordenadas, altitud, tipo de vegetación, fecha de colecta, método de colecta y colector; además de la información taxonómica: especie, descriptor y año de descripción, determinador y año. La mayoría de los ejemplares permanecen en alcohol al $70 \%$, algunos se montaron en seco. La información obtenida de las colectas y del proceso taxonómico se capturó en una hoja de Excel, además de la información de las etiquetas se capturó familia, subfamilia, tribu, género, castas y número de individuos. Para la lista comentada se elaboró una ficha por especie, con la siguiente información: nombre de la especie, autor y año; número de individuos colectados, castas; fecha de colecta y tipo de vegetación; distribución de la especie mundial y en México; Además de morfología, biología y distribución de cada género.

\section{RESULTADOS}

Se colectó un total de 4,815 individuos pertenecientes a 26 especies, 15 géneros, 12 tribus y 5 subfamilias (Cuadro 1). La subfamilia con mayor riqueza de especies fue Myrmicinae, con 13 especies; seguida de Formicinae con 7 especies; Dolichoderinae con tres especies; Dorylinae con dos especies; mientras Ponerinae es la que presenta la menor riqueza con sólo una especie. Además, 
se cuenta con ocho nuevos registros para el estado de Aguascalientes: Camponotus andrei, C. conspicuus zonatus, C. mina, Formica argentea, Myrmecocystus mexicanus, Neivamyrmex rugulosus, Crematogaster isolata y Trachymyrmex smithi.

Los géneros con mayor riqueza de especies fueron: Pheidole con seis especies; Camponotus con cuatro; Myrmecocystus, Neivamyrmex y Pogonomyrmex con dos cada uno; mientras que los géneros con menor riqueza de especies fueron Dorymyrmex, Forelius, Liometopum, Formica, Odontomachus, Crematogaster, Monomorium, Novomessor, Solenopsis y Trachymyrmex con una especie cada uno. La especie más abundante es Liometopum apiculatum con 1,887 individuos y la especie menos abundante fue Camponotus conspicuus zonatus con 2 individuos.

El tipo de vegetación con mayor riqueza de especies fue el bosque de encino con 20 especies (76.92\%), de las cuales 10 fueron exclusivas; mientras que la vegetación secundaria presentó 16 especies $(61.53 \%)$ de la cuales 6 fueron exclusivas; 10 especies estuvieron presentes en ambas vegetaciones. La vegetación de bosque de encino también tiene la mayor abundancia de individuos, 3,033 $(62.99 \%)$, mientras que la vegetación secundaria fue la menos abundante con 1,782 individuos (37\%).

La temporada con mayor riqueza fue verano con 24 especies (92\%) de las cuales 2 son exclusivas; en segundo lugar, fue primavera con 20 especies (77\%); en tercer lugar otoño con 16 especies (62\%) y finalmente invierno con 13 especies (50\%). Dos especies fueron exclusivas de una temporada, nueve especies de dos de ellas, seis especies de tres temporadas y nueve especies estuvieron en las cuatro temporadas muestreadas. En cuanto a la abundancia por temporada, verano presenta la mayor abundancia de individuos con, 3068 (63\%), seguido por otoño con 643 $(13.35 \%)$, primavera con $599(12.44 \%)$ mientras que invierno presento la menor abundancia con 505 individuos (10.48\%).

El método de colecta con mayor riqueza fueron las trampas cebadas, con 24 especies $(92.3 \%)$ de las cuales ocho son exclusivas; seguido por colecta directa, con 18 especies $(69.23 \%)$ de las cuales dos son exclusivas y por último el cernido de hojarasca con 3 especies (11.53\%). Diecinueve especies fueron colectadas con la trampa de caída con atún, dos de ellas exclusivas; 15 especies con necrotrampa, una de ellas exclusiva; 14 especies con trampa de caída con calamar y nuez de las cuales dos son exclusivas de la trampa de caída con nuez y sólo ocho especies con trampa de caída con fruta.

\footnotetext{
Lista comentada de las especies de hormigas del cerro Juan El Grande, EI Llano, Aguascalientes DOLICHODERINAE Forel, 1878

Dolichoderini Forel, 1878

Dorymyrmex Mayr, 1866

Las hormigas pertenecientes a este género se caracterizan
}

por la parte posterior del propodeo terminando en forma de cono; los palpos maxilares muy largos, con 6 segmentos. Todas las especies de Dorymyrmex construyen sus nidos en el suelo, en regiones áridas o semiáridas, prefiriendo los lugares abiertos, de escasa cobertura vegetal en donde suelen jugar un papel dominante desde el punto de vista ecológico. Distribución: desde el norte de Estados Unidos hasta el sur de Argentina (Cuezzo y Guerrero 2012, Cuezzo 2003).

\section{Dorymyrmex insanus (Buckley, 1866)}

Se colectaron 314 ejemplares: obreras; 267 en otoño, 10 en invierno, 14 en primavera y 23 en verano. En bosque de encino 24 y 290 en vegetación secundaria. Distribución: Neártica y Neotropical. Presente en los estados de Aguascalientes, Baja California, Baja California Sur, Chihuahua, Durango, Hidalgo, Nayarit, Nuevo León, Puebla, Querétaro, Quintana Roo, Sinaloa, Sonora, Tamaulipas, Veracruz y Yucatán (Vásquez-Bolaños 2015, Vásquez-Bolaños y Escoto-Rocha 2016).

\section{Forelius Emery, 1888}

Las hormigas pertenecientes a este género se caracterizan por el pronoto usualmente con 2 vellosidades rectas y largas, además de otras cortas; mandíbulas con 5 ó 6 dientes; pelos curvos colocados encima del clípeo. Las especies de Forelius anidan siempre en el suelo de ambientes desérticos o semidesérticos de escasa o nula cobertura vegetal. La manera más sencilla de reconocerlas es porque la mayoría de sus especies tienen el espiráculo propodeal alargado (dos o más veces más largo que ancho). Forrajeras generalistas. Distribución: disyunta con un grupo de especies desde el centro de Estados Unidos al centro de México y otro grupo exclusivamente sudamericano (Mackay y Mackay, 1989, Fernández 2003).

Forelius mccooki (McCook, 1880)

Se colectaron 145 ejemplares: obreras; 30 en otoño, 67 en invierno, 25 en primavera y 23 verano. En bosque de encino una y 144 en vegetación secundaria. Distribución: Neártica y Neotropical. Presente en los estados de Aguascalientes, Baja California, Baja California Sur, Colima, Durango, Hidalgo, Jalisco, Nayarit, Nuevo León, Puebla, Querétaro, Sinaloa, Sonora, Tabasco, Tamaulipas, Veracruz y Yucatán (Vásquez- Bolaños 2015, Vásquez-Bolaños y EscotoRocha 2016).

\section{Tapinomini Emery, 1913 \\ Liometopum Mayr, 1866}

Las hormigas pertenecientes a este género se caracterizan por el gáster cubierto con pubescencia gris, mesosoma vista de perfil sin impresión antes del propodeo, polimórficas, las obreras mayores con ocelos, mandíbula con dientes y dientecitos. Nidos usualmente en árboles o troncos de encinos en el norte del país. Las obreras despiden un olor ocre cuando son molestadas. Forrajeras generalistas. 
Distribución: Neártica, Paleártica y Oriental (Del Toro et al. 2009, Alatorre-Bracamontes y Vásquez-Bolaños 2010).

\section{Liometopum apiculatum Mayr, 1870}

Se colectaron 1,887 ejemplares: obreras; 16 en otoño, 5 en invierno, 124 en primavera y 1,742 verano. Todas en bosque de encino. Distribución: Neártica. Presente en los estados de Chihuahua, Coahuila, Colima, Durango, Guanajuato, Hidalgo, México, Michoacán, Nuevo León, Puebla, Querétaro, Quintana Roo, San Luis Potosí, Sinaloa, Tamaulipas, Tlaxcala, y Zacatecas (Vásquez-Bolaños 2015, Vásquez-Bolaños y Escoto-Rocha 2016).

FORMICINAE Latreille, 1809

Camponotini Forel, 1878

Camponotus Mayr, 1861

Género con tamaño variable, desde pequeñas hasta muy grandes. El margen posterior del clípeo está alejado de los alveólos antenales por una distancia igual o mayor al diámetro de estos alveólos. No hay abertura de la glándula metapleural. El mesosoma convexo, con variedad en las especies, sin espinas, con espinas y angulaciones, el propodeo también presenta variación, desde cuadrado hasta angulado en vista dorsal. Algunas obreras y soldados tienen la cabeza modificada para obstruir las entradas a los nidos. En general se consideran omnívoras con colonias de tamaño variable en el suelo, bases o copas de árboles. Cosmopolitas, se encuentra desde el nivel del mar hasta más de 3,000 metros (Mackay y Mackay 1989, Fernández 2003).

\section{Camponotus andrei Forel, 1885}

Se colectaron 20 ejemplares: 15 obreras y seis soldados; cinco en primavera y 15 en verano. Todas en bosque de encino; Distribución: Neotropical. Nuevo registro para el estado. Presente en los estados de Baja California, Colima, Durango, Guerrero, Guanajuato, Hidalgo, México, Morelos, Oaxaca, Sonora, Veracruz y Zacatecas (VásquezBolaños 2015).

\section{Camponotus atriceps (Smith, 1858)}

Se colectaron 201 ejemplares: 165 obreras y 36 soldados; 40 en otoño, 57 en invierno, 18 en primavera y 86 en verano. Todas en bosque de encino. Distribución: Neártica y Neotropical. Presente en los estados de Campeche, Ciudad de México, Guerrero, Hidalgo, Jalisco, Michoacán, Morelos, Nayarit, Nuevo León, Oaxaca, Puebla, Quintana Roo, San Luis Potosí, Sinaloa, Sonora, Tabasco, Tamaulipas, Tlaxcala, Veracruz y Yucatán (Vásquez-Bolaños 2015).

Camponotus conspicuus zonatus Emery, 1894

Se colectaron dos ejemplares: obreras; uno en primavera y uno en verano. En bosque de encino uno y uno en vegetación secundaria. Nuevo registro para el estado. Distribución: Neotropical. Presente en los estados de Campeche, Jalisco, Puebla, Quintana Roo y Tabasco
(Vásquez-Bolaños 2015).

Camponotus mina Forel, 1879

Se colectaron seis ejemplares: cuatro obreras y dos soldados; dos en primavera y cuatro en verano. Todas en bosque de encino. Nuevo registro para el estado. Distribución: Neártica. Presente en los estados de Baja California, Baja California Sur, Chihuahua, Hidalgo, Morelos y Sonora (Vásquez-Bolaños 2015, VásquezBolaños y Escoto-Rocha 2016).

Lasini Ashmead, 1905

Myrmecocystus Wesmael, 1838

Las hormigas pertenecientes a este género se caracterizan por poseer palpos maxilares muy largos, con el segmento número 4 tan largo como los segmentos 5 y 6 juntos. Psamóforo presente. Son las conocidas hormigas de la miel de las zonas áridas de EEUU y México. Poseen una casta, las llamadas "repletas", que almacenan sustancias parecidas a la miel para servir de consumo a los demás miembros de la colonia. Forrajeras generalistas, hormigas mieleras. Distribución: norte dentro del Estado de Washington, hasta el altiplano mexicano (Mackay y Mackay 1989, Fernández 2003).

\section{Myrmecocystus melliger Forel, 1886}

Se colectaron 23 ejemplares: obreras.; uno en otoño, dos en invierno, cinco en primavera y 15 en verano. En Bosque de encino dos y 21 a vegetación secundaria. Distribución: Neártica y Neotropical. Presente en los estados de Aguascalientes, Chihuahua, Ciudad de México, Durango, Guerrero, Guanajuato, Hidalgo, Jalisco, México, Michoacán, Nuevo León, Puebla, Querétaro, San Luis Potosí, Sonora y Zacatecas (Vásquez-Bolaños 2015, Vásquez-Bolaños y Escoto-Rocha 2016).

Myrmecocystus mexicanus Wesmael, 1838

Se colectaron cinco ejemplares: obreras; tres en invierno, uno en primavera y uno en verano. Todas en bosque de encino. Nuevo registro para el estado. Distribución: Neártica y Neotropical. Presente en los estados de Baja California, Baja California Sur, Coahuila, Chihuahua, Durango, Guanajuato, Hidalgo, México, Nuevo León, San Luis Potosí y Tamaulipas (Vásquez-Bolaños 2015).

Formicini Latreille, 1809

Formica Linnaeus, 1758

Las hormigas pertenecientes a este género son de tamaño mediano, área frontal bien definida, ocelos presentes, polimórficas, antenas de 12 segmentos, alvéolos antenales situados cerca al margen del clípeo, mandíbulas triangulares con 7 o más dientes. Estas hormigas son esclavistas; forrajeras generalistas, ocurren en casi todo México, especialmente en las montañas. Distribución: Neártica y Paleártica (Mackay y Mackay 1989, Fernández 2003, Alatorre-Bracamontes y Vásquez-Bolaños 2010). 
Formica argentea Wheeler, 1902

Se colectaron ocho ejemplares: siete obreras y un soldado. uno en primavera y siete en verano. Todas en bosque de encino. Nuevo registro de género para el estado. Distribución: Neártica. Presente en los estados de Baja California, Chihuahua y Veracruz (Vásquez-Bolaños 2015).

DORYLINAE Leach, 1815

Dorylini Leach, 1815

Neivamyrmex Borgmeier, 1940

Este género de hormiga se caracteriza por la ausencia de dientecillos en las uñas tarsales. Género más rico en especies de la subfamilia. Hormigas legionarias, nómadas, depredadores de un conjunto restringido de artrópodos Distribución: Neotropical, alcanzando el sur de la región Neártica (Mackay y Mackay 1989, Fernández 2003, Alatorre-Bracamontes et al. 2015).

Neivamyrmex rugulosus Borgmeier, 1953

Se colectaron 25 ejemplares: 21 obreras y cuatro soldados; seis en otoño y 19 en verano. Todos en bosque de encino. Nuevo registro para estado. Distribución: Neotropical y Neártica. Presente en los estados de Jalisco, Nayarit, Sonora y Veracruz (Vásquez-Bolaños 2015).

Neivamyrmex texanus Watkins, 1972

Se colectaron diez ejemplares: ocho obreras y dos soldados; en verano. Todos en bosque de encino. Distribución: Neotropical y Neártica. Presente en los estados de Chihuahua, Coahuila, Hidalgo, Jalisco, San Luis Potosí y Sonora (Vásquez-Bolaños 2015).

PONERINAE Lepeletier, 1835

Ponerini Lepeletier, 1835

Odontomachus Latreille, 1804

Las hormigas pertenecientes a este género se encuentran en bosques o hábitats ribereños, aunque también se encuentran en hábitats desérticos. Caracterizadas por la carina nucal en forma de V, con líneas oscuras en la parte media, nudo del pecíolo con ápice dorsal generalmente cónico o puntiagudo. Depredadoras agresivas (Mackay y Mackay 1989, Fernández 2003).

Odontomachus clarus Roger, 1861

Se colectaron seis ejemplares: obreras; solamente en verano. Todos en bosque de encino. Presente en los estados de Chihuahua, Ciudad de México, Coahuila, Colima, Guerrero, Guanajuato, Hidalgo, Jalisco, Michoacán, Nuevo León, Puebla, Querétaro, Sonora y Tamaulipas (AlatorreBracamontes y Vásquez-Bolaños 2010, Vásquez-Bolaños 2015).

\section{MYRMICINAE Lepeletier, 1835}

Attini Smith, 1858

Pheidole Westwood, 1839

Las hormigas pertenecientes a este género son comunes todo el mundo, caracterizadas por las antenas de 12 artejos con una maza clara de tres artejos. Mandíbulas con numerosos dientes y dentículos. Normalmente dimórficas o polimórficas, el soldado con cabeza dos veces más grande que el gáster. Habitantes principalmente del estrato epigeo (Mackay y Mackay 1989, Fernández 2003).

\section{Pheidole sp. 1}

Se colectaron 1,293 ejemplares: 1,176 obreras y 117 soldados; 207 en otoño, 63 en invierno, 268 en primavera y 755 en verano. En Bosque de encino 245 y 1048 a vegetación secundaria.

\section{Pheidole sp. 2}

Se colectaron 128 ejemplares: 118 obreras y diez soldados, tres en otoño, 124 en invierno y uno en verano. En Bosque de encino 48 y 80 en vegetación secundaria.

Pheidole sp. 3

Se colectaron 198 ejemplares: 181 obreras y 17 soldados; diez en otoño, 24 en primavera y 164 en verano. Todos en Bosque de encino.

\section{Pheidole sp. 4}

Se colectaron 22 ejemplares: soldados; uno en otoño y 21 en verano. En Bosque de encino uno y 21 en vegetación secundaria.

\section{Pheidole sp. 5}

Se colectaron 14 ejemplares: obreras; diez en primavera y cuatro en verano. Todos en vegetación secundaria.

\section{Pheidole sp. 6}

Se colectaron 14 ejemplares: obreras; tres en primavera $\mathrm{y}$ once en verano. Todos en vegetación secundaria.

\section{Trachymyrmex Forel, 1893}

Las hormigas pertenecientes a este género se caracterizan por poseer escrobos antenales distintos y pocos profundos. Cuerpo con numerosos tubérculos, incluyendo el primer segmento del gáster. Hormigas monomórficas. Usualmente de color oscuro. Especialistas de climas tropicales. Cultivadoras de hongos (Mackay y Mackay 1989, Fernández 2003).

\section{Trachymyrmex smithi Buren, 1944}

Se colectaron siete ejemplares: obreras; uno en invierno, uno en primavera y cinco en verano. Todos en vegetación secundaria. Nuevo registro para el estado. Distribución: Neártica. Presente en los estados de Coahuila y Chihuahua (Vásquez-Bolaños 2015). 
Crematogastrini Forel, 1893

Crematogaster Lund, 1831

Las hormigas pertenecientes a este género se caracterizan por poseer la inserción del pospecíolo con el gáster, el cual se articula con la superficie dorsal del tergo abdominal. En vista dorsal el gáster en forma de corazón. Hormigas arbóreas, algunas omnívoras de suelo. Mirmícinos cuyas especies son conocidas por atender homópteros. En los Estados Unidos, vulgarmente llamadas hormigas acróbatas, anidan en casas, forrajean día y noche y prefieren alimentos de alto contenido proteico (Mackay y Mackay 1989, Fernández 2003).

Crematogaster isolata Buren, 1968

Se colectaron 18 ejemplares: obreras; dos en otoño y 16 en primavera. Todos en vegetación secundaria. Distribución: Neártica. Presente en los estados de Chihuahua y Durango (Morgan y Mackay 2017).

Pogonomyrmecini Ward, Brady, Fisher y Schultz, 2014 Pogonomyrmex Mayr, 1868

Las hormigas pertenecientes a este género comunes en las regiones Neártica, tamaño mediano, superficie ventral de la cabeza usualmente con psamofora, el propodeo, usualmente presenta un par de espinas, espuelas pectinadas en las tibias medias y posteriores, aguijón bien desarrollado; hormigas principalmente en áreas áridas y semiáridas y de zonas abiertas. Granívoras y forrajeras generalistas. Distribución: Neotropical y Neártica (Mackay y Mackay 1989, Fernández 2003).

Pogonomyrmex barbatus (Smith, 1858)

Se colectaron 22 ejemplares: obreras; ocho en otoño y 14 en verano. En Bosque de encino 13 y nueve en vegetación secundaria. Distribución: Neártica y Neotropical. Presente en los estados de Aguascalientes, Chihuahua, Colima, Durango, Guerrero, Guanajuato, Hidalgo, Jalisco, México, Michoacán, Nayarit, Nuevo León, Oaxaca, Puebla, Querétaro, San Luis Potosí, Sonora, Tamaulipas, Tlaxcala, Veracruz y Zacatecas (Vásquez-Bolaños 2015, VásquezBolaños y Escoto-Rocha 2016).

Pogonomyrmex rugosus Emery, 1895

Se colectaron ocho ejemplares: obreras; tres en otoño, uno en invierno y cuatro en primavera. Todos en vegetación secundaria. Presente en los estados de Aguascalientes, Baja California, Chihuahua, Coahuila, Durango, Nayarit, Nuevo León, Sinaloa, Sonora, Tamaulipas y Zacatecas (VásquezBolaños 2015, Vásquez-Bolaños y Escoto-Rocha 2016).

Solenopsidini Forel, 1893

Monomorium Mayr, 1855

Las hormigas pertenecientes a este género son pequeñas a moderadas en tamaño, monomórficas a polimórficas, Antenas de 12 segmentos y una maza antenal compuesta por tres artejos, clípeo bicarinado, usualmente de color negro brillante. Algunas especies son plagas caseras distribuidas por las actividades humanas. Generalistas, especialistas, forrajeras. Distribución cosmopolita (Mackay y Mackay 1989, Fernández 2003).

Monomorium minimum (Buckley, 1867)

Se colectaron 213 ejemplares: obreras; 28 en otoño, siete en invierno, 53 en primavera y 125 en verano. En Bosque de encino 182 y 31 en vegetación secundaria. Distribución: Neártica. Presente en los estados de Aguascalientes, Baja California, Chihuahua, Coahuila, Durango, Hidalgo, Jalisco, Michoacán, Nayarit, Nuevo León, Querétaro, Sinaloa, Sonora, Tamaulipas, Veracruz y Zacatecas (Vásquez-Bolaños 2015, Vásquez-Bolaños y Escoto-Rocha 2016).

\section{Solenopsis Westwood, 1840}

Las hormigas pertenecientes a este género son pequeñas, monomóficas o dimórficas, las especies más comunes son dimórficas; antena con diez artejos y una maza antenal de dos; pronoto sin espinas; clípeo bicarinado. Omnívoras, depredadoras y forrajeras generalistas. Distribución cosmopolita (Mackay y Mackay 1989, Fernández 2003).

Solenopsis geminata (Fabricius, 1804)

Se colectaron 174 ejemplares: 166 obreras y ocho soldados; tres en otoño, 161 en invierno, tres en primavera y siete en verano. En Bosque de encino 150 y 24 a vegetación secundaria. Distribución: Neártica y Neotropical. Presente en los estados de Aguascalientes, Baja California, Campeche, Chiapas, Coahuila, Colima, Durango, Guerrero, Hidalgo, Jalisco, Morelos, Nuevo León, Puebla, Quintana Roo, Tabasco, Tamaulipas, Tlaxcala, Veracruz y Yucatán (Vásquez-Bolaños 2015, Vásquez-Bolaños y Escoto-Rocha 2016).

Stenammini Ashmead, 1905

Novomessor Emery, 1915

Hormigas de tamaño medio, usualmente monomórficas, con el tercer diente (contando desde el apical) de la mandíbula más grande que el cuarto; los escapos de las antenas llegan más allá del borde posterior de la cabeza; cuerpo delgado, con patas largas; psamofora ausente. Forrajeras generalistas. Distribución: Cosmopolitas (Mackay y Mackay 1989, Fernández 2003).

Novomessor cockerelli (André, 1893)

Se colectaron 52 ejemplares: obreras; 18 en otoño, cuatro en invierno, siete en primavera y 23 en verano. Todos en vegetación secundaria. Distribución: Neártica. Presente en los estados de Aguascalientes, Coahuila, Chihuahua, Durango, Nuevo León, Sonora y Zacatecas (VásquezBolaños 2015, Vásquez-Bolaños y Escoto-Rocha 2016). 


\section{DISCUSIÓN}

En el presente trabajo se colectaron 26 especies, de las cuales seis no fue posible determinar a nivel especie; estas morfoespecies pertenecen al género Pheidole, que es uno de los de mayor complejidad taxonómica por la gran cantidad de especies (Wilson 2003), lo que hace más difícil lograr su determinación, es necesario prestar más atención ya que se podrían encontrar nuevas especies o registros nuevos. El género Pheidole presentó la mayor riqueza de especies, seis, esto coincide con lo que se reporta en otros trabajos de hormigas (Hinojosa-Ontiveros et al. 2015, Bizuet-Flores et al. 2015), en donde el género Pheidole presenta mayor diversidad de especies, con 624 especies para América y 900 especies descritas en todo el mundo y esto gracias a que estas hormigas están presentes en una amplia distribución de hábitats (Wilson 2003). La subfamilia con la mayor riqueza de especies fue Myrmicinae, seguida de Formicinae mientras que las que presentaron menor riqueza fue Dorylinae y Ponerinae, esto coincide con lo que se reporta en otros trabajos de hormigas (Reynoso-Campos et al. 2015, Jaffé 1993, Salcido-López et al. 2012) lo que puede considerarse lógico ya que las primeras dos subfamilias son las más diversas en especies en México y en el mundo, mientras que las subfamilias menos diversas coinciden ya que estas dos subfamilias Dorylinae y Ponerinae son las menos diversas para México (Vásquez-Bolaños 2015, Rojas-Fernández 2001). Con respecto a la subfamilia con mayor abundancia fue Dolichoderinae seguido por Myrmicinae, siendo la subfamilia Dolichoderinae cuyas colonias pueden estar formadas por unos pocos centenares de individuos a varios miles, además de que estas dos subfamilias son de las más abundantes en todo el mundo (Cuezzo 2003, Reynoso-Campos et al. 2015) mientras que la subfamilia Ponerinae presento la menor abundancia con seis individuos, cuyas colonias de estos van desde una docena hasta cientos o escasos miles de individuos (Vásquez Bolaños 1998).

Liometopum apiculatum es una especie que establece trayectos de forrajeo, las obreras son procuradoras de alimento fuera del nido, son omnívoras, el tipo de vegetación donde comúnmente se encuentra son en bosques de encino (Quercus spp.) y la población de hormigas en los nidos van desde unos pocos cientos hasta 82,500 obreras (Lara-Juárez et al. 2015), probablemente esto se deba a que esta especie fue la más abundante. El bosque de encino presentó la mayor riqueza, 20 especies, con diez exclusivas; mientras que la vegetación secundaria presentó la menor riqueza, con 16 especies, siendo esta una área de disturbio por las actividades de pastoreo de ganado, lo que se observa claramente es que el número de especies decrece a medida que el grado de perturbación aumenta, además que el BE resulta ser la vegetación con el mayor número de especies; esto pudiera deberse a que presentara mayor cobertura vegetal además de contar con hojarasca en los suelos en cambio la VS presentaba un suelo desnudo y poca cobertura vegetal, lo que concuerda con Rojas-Fernández (2001), en donde menciona que en lugares perturbados existe menor diversidad de especies, además la gran mayoría de las hormigas se encuentran viviendo dentro de las ramas y trozos pequeños de madera muerta y entre o debajo de la capa de hojarasca, mientras que muy pocas construyen su nido directamente en el suelo. En el caso de abundancia por tipo de vegetación se encontró un mayor número de individuos en el BE que en la VS lo contrario con lo reportado por Rojas-Fernández (2001) en donde menciona que los sitios perturbados se observa una mayor abundancia y biomasa de hormigas que en los sitios sin perturbar, esto se le puede atribuir a que la mayoría de la especies con mayor abundancia encontradas solo se encuentran asociados a BE como es el caso de la especie Liometopum apiculatum, aunque también pudo haberse afectado por la mayor disponibilidad de recursos que pueden ser utilizados por las hormigas.

Durante el tiempo de colecta la temporada que tuvo mayor riqueza y abundancia fue verano, mientras que invierno fue que presentó la menor riqueza y abundancia de especies, cabe destacar que en verano es temporada de lluvias, mientras que en la temporada de invierno en donde se presenta los días con mayores heladas (INEGI 2001, 2016). Esto concuerda con Kaspari (2003) en donde menciona que los factores bióticos como interacción entre poblaciones, así como la disponibilidad de recursos y los factores abióticos como temperatura y humedad son muy importantes para la productividad de las poblaciones de hormigas, de estos quizás la temperatura es la más importante dado que las hormigas regulan su temperatura a partir de la temperatura ambiental. Según Hölldobler y Wilson (1990) están limitadas para forrajear si no hace mucho calor ni mucho frío. Razón por la cual con base en la temperatura es probable que haya disminuido la riqueza de especies y la abundancia en invierno. En cuanto a la humedad, Schumacher y Whitford (1976) mencionan que la baja humedad también puede limitar el forrajeo; además de que el número de hormigas frecuentemente parece estar dado por la lluvia ya que están relacionado con el incremento de cobertura vegetal además de ser un buen predictor de la producción de semillas de las cuales se alimentan las hormigas (Morton y Davidson 1988), lo que parece apoyar como explicación del incremento a la riqueza de especies con la tendencia dado por las lluvias. La mayoría de las especies de hormigas fueron colectadas con trampas cebadas (tomando en cuanta NTP-80), con 24 (equivalente al 92\% del total) siendo este método el más eficiente, este resultado concuerda con Sarmiento-M. (2003) y Silvestre et al. (2003) ya que las especies más propensas a visitar fuentes artificiales de alimento (cebos) son especies generalistas, las cuales generalmente exploran cualquier nuevo tipo de recurso disponible en su área de forrajeo, esto parece lógico ya que la mayoría de especies colectadas tanto por este método, como en general fueron especies forrajeras generalistas, además de que las trampas de caída cebadas son de los mejores métodos 
que proponen para colecta de hormigas. Hay dos especies que sólo se colectaron de manera directa, Crematogaster isolata y Neivamyrmex texanus, siendo esta última especie depredadoras de otras hormigas, por lo que también el método de colecta directa es el más indicado para tener un cubrimiento completo de la riqueza de hormigas en un lugar, por lo que es más recomendable aplicar varios métodos de colecta, ya que esto permite colectar hormigas de diferentes hábitos (Sarmiento-M. 2003).

Comparando estos resultados con otro estudio de hormigas que utilizan métodos de colecta similar se obtuvo un número de especies menor al reportado en el trabajo de Salcido-López et al. (2012) en donde se estudió la mirmecofauna en el cerro La Culebra, Arandas, Jalisco, en donde reportan 32 especies, esto tal vez de deba a la poca variedad en tipos de vegetación del cerro Juan El Grande además del nivel de disturbio por parte del pastoreo de ganado o la frecuente presencia de personas, lo que desfavorece a la mirmecofauna; aunque se obtuvo similitud con respecto a la riqueza de especies en el tipo de vegetación. En cuanto al método de colecta, resultó con una mayor riqueza de especies el presente trabajo en comparación con Cañedo-Ortiz (1988) en Aguascalientes quien reporta 11 especies, esto probablemente se deba a que sólo se implemento un único método de colecta. Las desventajas en este trabajo de cada tipo de metodología fueron: las trampas de caída cebadas pueden ser dañadas por animales, que es el caso de la vegetación secundaria al ser un área perturbada por ganado fueron estropeadas algunas de ellas, el terreno es una principal limitante ya que estas trampas deben ir enterradas en el suelo y en algunas áreas el terreno fue difícil de excavar tanto por los suelos rocosos así como por las raíces de algunas plantas y no garantiza la captura de diferentes castas. La colecta directa requiere tiempo y paciencia para la localización de las hormigas y algunas huyen rápidamente, además del tamaño, lo que dificulta la colecta en lugares de difícil acceso o lugares recónditos. Los sacos Winkler sólo van dirigidos a la captura de hormigas del suelo y habitantes casi exclusivos de la hojarasca. Algunos de los problemas relacionados con los tipos de colecta los reporta RodríguezGarza (2015). De las especies encontradas en el cerro Juan El Grande existen especies de suma importancia, en donde destacan Solenopsis geminata denominada "hormiga fuego" estas hormigas son capaces de deformar las plantas, además puede causar serios problemas a la salud humana produciendo reacciones alérgicas por el veneno y la dolorosa picadura (Ulloa-Chacón 2003); Liometopum apiculatum, Myrmecosistus melliger y $M$. mexicanus son hormigas de importancia económica en México, ya que en algunos estados de la República Mexicana se colectan para consumo humano, en el caso de L. apiculatum son consumidas las pupas llamadas popularmente escamoles (Lara-Juárez et al. 2015) además de Pogonomyrmex barbatus son de las hormigas de granívoras más importantes y especializadas que remueve grandes cantidades de semillas del suelo
(Ramírez-Lucas y Ríos-Casanova 2015). En el presente estudio las especies de hormigas colectadas representan el $50 \%$ de la mirmecofauna total registrada para el estado y $2.1 \%$ de las especies registradas de esta familia para México (Vásquez-Bolaños y Escoto-Rocha 2016, VásquezBolaños 2015). También se contribuye a nivel estado pues se tienen 8 nuevos registros para Aguascalientes, con lo cual se incrementa la riqueza a 40 especies, en donde se tenían 32 (Vásquez-Bolaños y Escoto-Rocha 2016). Cabe destacar que con este trabajo pasa de ser el municipio de El Llano con nulos registros de hormigas al de mayor número de especies registradas para el estado. Realizar más estudios relacionados con las hormigas en el estado de Aguascalientes ya que son escasos los estudios de hormigas, a fin de contar con listados lo más completos posibles sobre la diversidad de estos organismos. Realizar más colectas en los municipios que no se tiene registros de hormigas para el estado además de implementar más metodologías a fin de encontrar posibles nuevos registros. Se recomienda trabajar más con taxonomía de Formicidae en México, especialmente en géneros con gran complejidad como lo es Pheidole.

\section{AGRADECIMIENTOS}

Este trabajo se dedica al Dr. Jorge Llorente, por su invaluable labor en el campo de la entomología en general, y de la lepidopterología en particular. Se agradece al M. en C. Jaime Escoto Rocha y al Dr. Jaime Antonio Escoto Moreno por el apoyo en las asesorías. El primer autor agradece infinitamente a sus padres Mario González Rangel, Margarita Martínez Cruz y abuela Esther Cruz García; a compañeros y colegas quienes apoyaron en el trabajo de campo Susana Rodríguez, Andrés Mares, Ariana Martínez, Jorge Eduardo Gutiérrez, José Luis Reyes y Ricardo Rivera.

\section{LITERATURA CITADA}

Alatorre-Bracamontes, C.E. y M. Vásquez-Bolaños, M. 2010. Lista comentada de las hormigas (Hymenoptera: Formicidae) del norte de México. Dugesiana, 17(1): 9-36.

Alatorre-Bracamontes, C.E., M. Vásquez-Bolaños, G. Castaño-Meneses y J.L. Navarrete-Heredia. 2015. La subfamilia Dorylinae (Hymenoptera: Formicidae) en la Colección Entomológica, Sección Formicidae, del Centro de Estudios en Zoología, Universidad de Guadalajara (CZUG). (pp. 41-51). In: Castaño-Meneses, G., M. Vásquez-Bolaños, J.L. Navarrete-Heredia, G.A. Quiroz-Rocha e I. Alcalá-Martínez (Coords.). Avances de Formicidae de México. Astra Editores, Guadalajara, Jalisco, México.

Alonso, L.E. y D. Agosti. 2000. Biodiversity studies, monitoring, and ants: an overview. (pp. 1-8). In: Agosti, D., J.D. Majer, L.E. Alonso y T.R. Shultz (Eds.). Ants, standard methods for measuring and monitoring biodiversity. Smithsonian Institution Press. Washington. 
Bolton, B. 1994. ldentification guide to the ant genera of the world. Harvard University Press, Cambridge.

Bizuet-Flores, M.Y., M. Vásquez-Bolaños y P.C. Martínez del Río. 2015. Variación espacial y temporal de hormigas (Hymenoptera: Formicidae) en la vegetación y humedales del Desierto Chihuahuense. (pp. 151-160). In: Castaño-Meneses, G., M. Vásquez-Bolaños, J.L. Navarrete-Heredia, G.A. Quiroz-Rocha e I. AlcaláMartínez (Coords.). Avances de Formicidae de México. Astra Editores, Guadalajara, Jalisco, México.

Cañedo-Ortiz, B.O. 1988. Distribución, biología $e$ implicaciones ecológicas y económicas de la entomofauna de la familia Formicidae en el municipio de Aguascalientes. Tesis de Licenciatura. Universidad Autónoma de Aguascalientes, Aguascalientes, Aguascalientes, México.

Castaño-Meneses, G., M. Vásquez-Bolaños, J.L. NavarreteHeredia, G.A. Quiroz-Rocha e I. Alcalá-Martínez (Coords.). 2015. Avances de Formicidae de México. Astra Editores, Guadalajara, Jalisco, México.

Cuezzo, F. 2003. Subfamilia Dolichoderinae. (pp. 291-297). In: Fernández, F. (Ed.). Introducción a las hormigas de la región Neotropical. Instituto de Investigación de Recursos Biológicos Alexander von Humboldt, Bogotá, Colombia.

Cuezzo, F. y R.J. Guerrero. 2012. The ant genus Dorymyrmex Mayr (Hymenoptera: Formicidae: Dolichoderinae) in Colombia. Psyche, 1-24. Doi:10.1155/2012/516058

Del Toro, I., J.A. Pacheco and W.P. MacKay. 2009. Revision of the ant genus Liometopum. Sociobiology, 53: 299369.

Escoto-Rocha, J., H.J. Cruz-Gutiérrez y L. DelgadoSaldivar. 2001. Biodiversidad de Himenópteros del estado de Aguascalientes. Investigación y Ciencia de la Universidad Autónoma de Aguascalientes, 24: 20-27.

Fernández, F. (Ed.). 2003. Introducción a las hormigas de la región Neotropical. Instituto de Investigación de Recursos Biológicos Alexander von Humboldt, Bogotá, Colombia.

Francoeur, A. 1973. Révision taxonomique des espèces néarctiques $\mathrm{du}$ groupe fusca, genre Formica (Formicidae, Hymenoptera). Mémoires de la Société entomologique du Québec, 3: 1-316.

Hinojosa-Ontiveros, G., M.P. González-Castillo y D. Ramírez-Noya. 2015. Listado de hormigas (Hymenoptera: Formicidae) de cuatro municipios del estado de Durango. (pp. 89-99). In: Castaño-Meneses, G., M. Vásquez-Bolaños, J.L. Navarrete-Heredia, G.A. Quiroz-Rocha e I. Alcalá-Martínez (Coords.). Avances de Formicidae de México. Astra Editores, Guadalajara, Jalisco, México.

Hölldobler, B. y E.O. Wilson. 1990. The Ants. Harvard University Press, Cambridge.

Instituto Nacional de Estadística, Geográfica e Informática (INEGI). 2001. Cuaderno estadístico del municipio de El Llano, Aguascalientes 2001. INEGI, México.
Instituto Nacional de Estadística, Geográfica e Informática (INEGI). 2009. Prontuario de información geográfica municipal de los Estados Unidos Mexicanos El Llano, Aguascalientes. INEGI, México.

Instituto Nacional de Estadística, Geográfica e Informática (INEGI). 2015. Anuario estadístico y geográfico de Aguascalientes 2015. INEGI, México.

Instituto Nacional de Estadística, Geográfica e Informática (INEGI). 2016. Anuario estadístico y geográfico de Aguascalientes 2016. INEGI, México.

Jaffé, K. 1993. El mundo de las hormigas. Equinoccio Ediciones-Universidad de Simón Bolivar, Caracas.

Kaspari, M. 2003. Introducción a la ecología de las hormigas. (pp. 97-108). In: Fernández, F. (Ed.). Introducción a las hormigas de la región Neotropical. Instituto de Investigación de Recursos Biológicos Alexander von Humboldt, Bogotá, Colombia.

Lara-Juárez, P., J.R. Aguirre-Rivera, P. Castillo-Lara y J.A. Reyes-Agüero. 2015. Biología y aprovechamiento de la hormiga de escamoles, Liometopum apiculatum (Hymenoptera: Formicidae). Acta Zoológica Mexicana, 31(2): 251-264.

MacGown, J.A., B. Boudinot, M. Deyrup and D.M. Sorger. 2014. A review of the Nearctic Odontomachus (Hymenoptera: Formicidae: Ponerinae) with a treatment of the males. Zootaxa, 3802(4): 515-552.

Mackay, W.P. y E.E. Mackay. 1989. Clave para los géneros de hormigas en México (Hymenoptera: Formicidae). (pp. 1-82). In: Quiroz, L.N. y L.M.P. Garduño (Eds.). Memorias II Simposio Nacional de Insectos Sociales. Oaxtepec, Morelos.

Morgan, C. E. y W. P. Mackay. 2017. The North American acrobat ants of the hyperdiverse genus Crematogaster (hymwoptera: Formicidae). Lambert Academic Publishing, USA.

Morton, S.R. and D.W. Davidson. 1988. Comparative structure of harvester ants communities in arid Australia and North America. Ecological Monographs, 58: 1938.

Norton, E. 1875. Notas sobre las hormigas mexicanas. $L a$ Naturaleza, 3: 179-189.

Rabeling, C., S.P. Cover, R.A. Johnson and U.G. Mueller. 2007. A review of the North American species of the fungus-gardening ant genus Trachymyrmex (Hymenoptera: Formicidae). Zootaxa, 1664: 1-53.

Ramírez-Lucas, I.L. y L. Ríos-Casanova. 2015. Semillas removidas por la hormiga Pogonomyrmex barbatus (Smith): especies, familias y formas de vida. (pp. 129138). In: Castaño-Meneses, G., M. Vásquez-Bolaños, J.L. Navarrete-Heredia, G.A. Quiroz-Rocha e I. AlcaláMartínez (Coords.). Avances de Formicidae de México. Astra Editores, Guadalajara, Jalisco, México.

Reynoso-Campos, J.J., J.A. Rodríguez-Garza y M. Vásquez-Bolaños. 2015. Hormigas (Hymenoptera: Formicidae) de la Isla Cozumel, Quintana Roo, México. (pp. 27- 39). In: Castaño-Meneses, G., M. Vásquez- 
Bolaños, J.L. Navarrete-Heredia, G.A. Quiroz-Rocha e I. Alcalá-Martínez (Coords.). Avances de Formicidae de México. Astra Editores, Guadalajara, Jalisco, México.

Ríos-Casanova, L., A. Valiente-Banuety V. Rico-Gray. 2004. Las hormigas del Valle de Tehuacán, (Hymenoptera: Formicidae): una comparación con otras zonas áridas de México. Acta Zoológica Mexicana (nueva serie), 20 (1): 37-54.

Rodríguez-Elizalde, I. de J. y J. Escoto-Rocha. 2015. Hormigas (Hymenoptera: Formicidae) de la Colección Zoológica del Departamento de Biología de la Universidad Autónoma de Aguascalientes. (pp. 61-70). In: Castaño-Meneses, G., M. Vásquez-Bolaños, J.L. Navarrete-Heredia, G.A. Quiroz-Rocha e I. AlcaláMartínez (Coords.). Avances de Formicidae de México. Astra Editores, Guadalajara, Jalisco, México.

Rodríguez-Garza, J.A. 2015. Hormigas (Hymenoptera: Formicidae) encontradas en la reserva de San Felipe Bacalar, Quintana Roo, México. (pp. 71-82). In: Castaño-Meneses, G., M. Vásquez-Bolaños, J.L. Navarrete-Heredia, G.A. Quiroz-Rocha e I. AlcaláMartínez (Coords.). Avances de Formicidae de México. Astra Editores, Guadalajara, Jalisco, México.

Rojas-Fernández, P. 1996. Formicidae (Hymenoptera). (pp.483-500). In: Llorente-Bousquets, J., A.N. GarcíaAldrete y E. González-Soriano (Eds.). Biodiversidad, taxonomía y biogeografía de artrópodos de México: Hacia una síntesis de su conocimiento. CONABIO, México, D. F.

Rojas-Fernández, P. 2001. Las hormigas del suelo en México: Diversidad, distribución: e importancia (Hymenoptera: Formicidae). Acta Zoológica Mexicana (n. s.), Número especial 1: 189-238.

Ulloa-Chacón, P. 2003. Hormigas urbanas. (pp. 351-359). In: Fernández, F. (Ed.). Introducción a las hormigas de la región Neotropical. Instituto de Investigación de Recursos Biológicos Alexander von Humboldt, Bogotá, Colombia.

Salcido-López, A., M. Vásquez-Bolaños y G.A. QuirozRocha. 2012. Hormigas (Hymenoptera: Formicidae) del cerro de la Culebra, Arandas, Jalisco, México. Dugesiana, 19(2): 151-155.

Recibido: 5 de junio 2019

Aceptado: 19 de junio 2019
Sarmiento-M., C. E. 2003. Metodología de captura y estudio de las hormigas. (pp. 201-210). In: Fernández F. (Ed.). Introducción a las hormigas de la región Neotropical. Instituto de Investigación de Recursos Biológicos Alexander von Humboldt, Bogotá, Colombia.

Schumacher, A. and W.G. Whitford. 1976. Spatial and temporal variation in Chihuahuan desert ant faunas. Southwestern Naturalist, 21:1-8.

Silvestre, R., C.R. Brandão y R. Rosa da Silva. 2003. Grupos funcionales de hormigas: el caso de los gremios del Cerrado. (pp. 113-148) In: Fernández, F. (Ed.). Introducción a las hormigas de la región Neotropical. Instituto de Investigación de Recursos Biológicos Alexander von Humboldt, Bogotá, Colombia.

SUBECO. 2001. Reporte sobre la comparación de la vegetación de 7 áreas prioritarias para la conservación en el Estado de Aguascalientes. Reporte interno de la Subsecretaría de Ecología. SEDESO. Gobierno del Estado de Aguascalientes, México.

Vásquez Bolaños, M. 1998. Hormigas (Hymenoptera: Formicidae) colectadas en necrotrampas, en tres localidades de Jalisco, México. Tesis de Licenciatura, Division de Ciencias Biológicas y Ambientales, Centro Universitario de Ciencias Biológicas y Agropecuarias, Universidad de Guadalajara. Zapopan, Jalisco.

Vásquez-Bolaños, M. 2011. Lista de especies de hormigas (Hymenoptera: Formicidae) para México. Dugesiana, 18(1):93-133.

Vásquez-Bolaños, M. 2015. Taxonomía de Formicidae (Hymenoptera) para México. Métodos en Ecología y Sistemática, 10(1):95-133.

Vásquez-Bolaños, M., G. Castaño-Meneses, A. CisnerosCaballero, G.A. Quiroz-Rocha y J.L. Navarrete-Heredia (Eds.). 2013. Formicidae de México. Orgánica Editores, Guadalajara, México.

Vásquez-Bolaños, M. y A. Cisneros-Caballero. 2013. Hormigas (Hymenoptera: Formicidae) de Aguascalientes. (pp.155). In: Memorias del XXI Congreso Nacional de Zoología. Aguascalientes, Aguascalientes, México.

Vásquez-Bolaños, M. y J. Escoto-Rocha. 2016. Hormigas (Hymenoptera: Formicidae) de Aguascalientes. Investigación y Ciencia de la Universidad Autónoma de Aguascalientes, 68: 6-4.

Vásquez-Bolaños, M. y G.A. Quiroz-Rocha. 2013. Introducción. (pp. 7-22). In: Vásquez-Bolaños, M., G. Castaño-Meneses, A. Cisneros-Caballero, G.A QuirozRocha y J.L. Navarrete-Heredia (Eds.). Formicidae de México. Orgánica Editores, Guadalajara, México.

Wilson, E.O. 1971. The Insect Societies. The Belknap Press of Harvard University Press, Cambridge.

Wilson, E.O. 2003. La hiperdiversidad como fenómeno real: el caso de Pheidole. (pp. 363). In: Fernández, F. (Ed.). Introducción a las hormigas de la región Neotropical. Instituto de Investigación de Recursos Biológicos Alexander von Humboldt, Bogotá, Colombia. 
Cuadro 1. Lista de especies de hormigas del Cerro Juan el Grande por método de colecta y tipo de vegetación. T=Trampas de caída, $\mathrm{D}=$ Colecta directa, $\mathrm{C}=$ Cernido de hojarasca. $\mathrm{BE}=$ Bosque de encino, $\mathrm{VS}=$ Vegetación secundaria.

$$
\text { Colecta Vegetación Ponerinae }
$$

Subfamilia / especie

$$
\text { T D } \quad \mathrm{C} \quad \mathrm{BE} \quad \mathrm{VS}
$$

Odontomachus clarus

Dolichoderinae

Myrmicinae

Dorymyrmex insanus

Forelius mccooki

Liometopum apiculatum

$\begin{array}{lllll}x & x & x & x & x\end{array}$

Crematogaster isolata

Monomorium minimum

$\mathrm{x} \quad \mathrm{x}$

$\mathrm{X} \quad \mathrm{X}$

Novomessor cockerelli

Formicinae

$\begin{array}{llll}\mathrm{x} & \mathrm{x} & \mathrm{x} & \mathrm{x}\end{array}$

Pheidole sp. 1

Camponotus andrei

Camponotus atriceps

$\mathrm{x} \quad \mathrm{X}$

$\mathrm{x}$

Pheidole sp. 2

Pheidole sp. 3

Camponotus conspicuus zonatus

X $\quad \mathrm{X}$

$\mathrm{X}$

Pheidole sp. 4

Camponotus mina

$\begin{array}{llll}\mathrm{X} & \mathrm{X} & \mathrm{X}\end{array}$

Formica argentea

Myrmecocystus melliger

\section{$\mathrm{x}$}

$\mathrm{x}$

Pheidole sp. 5

x $\quad \mathrm{x}$

X $\quad \mathrm{x}$

$\mathrm{x}$

$\mathrm{x}$

Pheidole sp. 6

Myrmecocystus mexicanus

Dorylinae

Neivamyrmex rugulosus

Neivamyrmex texanus
$\mathrm{X}$

$\mathrm{X}$
Pogonomyrmex barbatus

Pogonomyrmex rugosus

Solenopsis geminata

\begin{tabular}{|c|c|c|c|}
\hline $\mathrm{x}$ & & & $\mathrm{x}$ \\
\hline $\mathrm{x}$ & $\mathrm{x}$ & & \\
\hline $\mathrm{x}$ & $\mathrm{x}$ & $\mathrm{x}$ & $\mathrm{x}$ \\
\hline $\mathrm{x}$ & & & $\mathrm{x}$ \\
\hline $\mathrm{x}$ & & & $\mathrm{x}$ \\
\hline $\mathrm{x}$ & $\mathrm{x}$ & & $\mathrm{x}$ \\
\hline $\mathrm{x}$ & & & \\
\hline $\mathrm{x}$ & & & \\
\hline $\mathrm{x}$ & $\mathrm{x}$ & & $\mathrm{x}$ \\
\hline $\mathrm{x}$ & $\mathrm{x}$ & & \\
\hline $\mathrm{x}$ & $\mathrm{x}$ & & $\mathrm{x}$ \\
\hline $\mathrm{x}$ & $\mathrm{x}$ & & \\
\hline
\end{tabular}

Trachymyrmex smithi

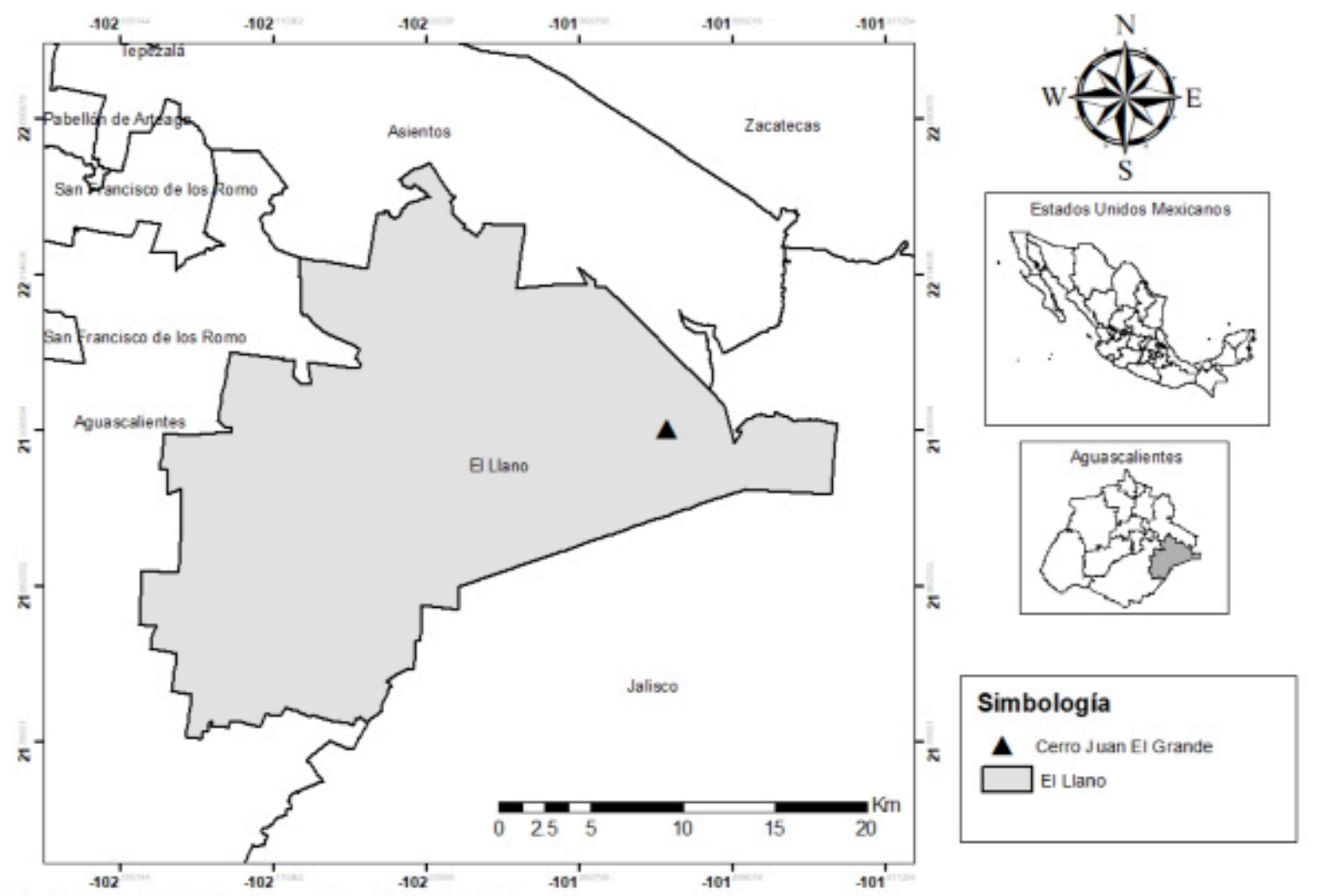

Q. José Luis Reyes-Hernández y Emmanuel Gonzalez-Martinaz(2017)

Figura 1. Ubicación del Cerro Juan El Grande, municipio El Llano, Aguascalientes. 